\title{
Quality of life measured by the St George's Respiratory Questionnaire and spirometry
}

\author{
M. Weatherall*, S. Marsh" ${ }^{\#}$ P. Shirtcliffe ${ }^{\#}$, M. Williams ${ }^{\#}$, J. Travers $^{\#}$ and R. Beasley
}

ABSTRACT: The present authors aimed to determine if the criteria for the diagnosis of chronic obstructive pulmonary disease (COPD) and its classification by severity as recommended by the Global Initiative for Chronic Obstructive Lung Disease are supported by measurements of respiratory health-related quality of life.

A community-based sample of adults aged 25-75 yrs had pre- and post-bronchodilator spirometry and completed the St George's Respiratory Questionnaire (SGRQ). Loess scatter plot smoothers of the SGRQ versus post-bronchodilator forced expiratory volume in one second (FEV 1 )/forced vital capacity (FVC) ratio and post-bronchodilator FEV $_{1} \%$ predicted together with receiver operating characteristic $(\mathrm{ROC})$ curve analysis were used to determine the relationship between spirometric variables and clinically important differences in the SGRQ score.

The scatter plot smoother and ROC curve analyses supported the value of 0.7 for postbronchodilator $\mathrm{FEV}_{1} / \mathrm{FVC}$ ratio, which was $\sim 4$ units higher than the nadir of the SGRQ. To represent a distance of 8 units on the SGRQ, the cut-off points for post-bronchodilator FEV 1 that delimit COPD severity stages were 80,60 and $40 \%$ pred for mild, moderate and severe COPD, respectively.

To diagnose chronic obstructive pulmonary disease the use of post-bronchodilator forced expiratory volume in one second/forced vital capacity ratio of 0.7 is supported by health-related quality of life measurements. There may be advantages in using forced expiratory volume in one second cut-off points of 80,60 and $40 \%$ predicted for the classification of mild, moderate and severe chronic obstructive pulmonary disease, respectively, similar to the approach recommended for asthma.

KEYWORDS: Chronic obstructive pulmonary disease, quality of life, spirometry

$\mathbf{T}$ he Global Initiative for Chronic Obstructive Lung Disease (GOLD) defines chronic obstructive pulmonary disease (COPD) as a post-bronchodilator forced expiratory volume in one second (FEV1)/forced vital capacity (FVC) ratio of $<0.7$, and not explained by another lung disease [1]. However, use of this fixed ratio has been criticised for not reflecting physiological changes of this variable with aging, potentially leading to the overdiagnosis of COPD, particularly in the elderly [2-5].

The utility of spirometry-based definitions of COPD is supported if other measurements of the impact of lung disease show a definite change in relation to spirometry. This constitutes a form of external validity for whatever measurement of lung function is chosen, be it FEV1, FEV1 \% predicted or absolute definitions based on the FEV1/FVC ratio. The St George's Respiratory Questionnaire (SGRQ), which is a measurement

For editorial comments see page 953. of respiratory quality of life, has been validated in COPD [6-8]. However, it was developed prior to the GOLD guidelines with their rigorous spirometric definition of COPD. Furthermore, these guidelines include severity cut-off criteria based on FEV1 \% pred, which are acknowledged to be based on simplicity rather than clinical validation [1]. Large longitudinal epidemiological studies would be required to validate the choice of such severity cut-off criteria; however, corroboration of these with meaningful differences in health-related quality of life could potentially serve as a useful surrogate for this. For the purposes of randomised controlled trials, a change in the total score for the SGRQ that represents a clinically important difference is 4 units [6]

The present study aims to examine if the value for the FEV1/FVC ratio of 0.7 can be supported by the measurement of respiratory quality of life in the SGRQ. Secondly, the current authors
AFFILIATIONS

*University of Otago Wellington, and ${ }^{*}$ Medical Research Institute of New Zealand, Wellington, New Zealand.

CORRESPONDENCE

R. Beasley

Medical Research Institute of

New Zealand

PO Box 10055

Wellington 6143

New Zealand

Fax: 6444729224

E-mail: Richard.Beasley@

mrinz.ac.nz

Received:

July 302008

Accepted after revision:

November 282008

STATEMENT OF INTEREST

None declared. 
explore whether the boundaries for severity of COPD based on the GOLD criteria, using cut-off points of FEV1 \% pred, are supported by clinically important quality of life changes reflected in the SGRQ.

\section{METHODS}

\section{Subjects}

Participants $(n=3,500)$ in the Wellington Respiratory Survey (WRS), were randomly selected from the electoral register, equally distributed by sex across the five-decade age groups from 25 to 75 yrs [9]. Subjects were sent a simple postal questionnaire seeking demographic, respiratory and smoking history data. All subjects who completed and returned questionnaires were invited to undertake more detailed questionnaires, pulmonary function tests and computed tomography scanning, as previously described in detail [9]. Subjects who completed all investigative modules formed the study group for the present analysis. The reference range population comprised 212 subjects who were never-smokers with no diagnosis of respiratory disease, no recent respiratory symptoms and no use of inhaled medication. The survey was approved by the Wellington Ethics Committee (Wellington, New Zealand) and written informed consent was obtained from each subject.

\section{Pulmonary function testing}

Pulmonary function tests in the WRS have been described in detail elsewhere $[9,10]$. In brief, spirometry measurements were carried out according to American Thoracic Society guidelines [11], a minimum of three acceptable manoeuvres were carried out and the best FEV1 and FVC results were selected for analysis. Spirometry was repeated $45 \mathrm{~min}$ after the administration of $400 \mu \mathrm{g}$ of salbutamol (Ventolin; GlaxoSmithKline NZ Ltd, Auckland, New Zealand) via a spacer (Space Chamber; Medical Developments International Ltd, Springvale, Australia). FEV1 and FVC values were expressed as \% pred of normal values [10].

\section{Quality of life questionnaire}

The SGRQ is a disease specific quality of life assessment tool validated in both COPD and asthma [12-14]. The questionnaire consists of 76 items divided into three parts measuring symptoms, activity limitation and social and emotional impact of disease. Each item is accorded a weight determined by the degree of distress accorded to each symptom or state described. Overall scores range from 0 (no effect on quality of life) to a maximum score of 100 (maximum perceived distress); thus, a higher score means a poorer quality of life and the questionnaire is suitable for administration in healthy people [8]. The English for New Zealand version was self completed by the subjects during the $45 \mathrm{~min}$ bronchodilation time. Results were entered into a spreadsheet, supplied by the questionnaire producers and pre-programmed with formulae to calculate a total score and scores for each of the individual components of the SGRQ (symptoms, activity and impacts). A change in the total score for the SGRQ that represents a clinically important difference is 4 units [6].

\section{Statistical methods}

Quantile regression [15] was used to find a formula to predict the upper $90 \%$ and $95 \%$ quantiles for the SGRQ based on age.
The dataset used for this was the 178 out of 212 subjects from a reference range dataset, who had values for the total score on the SGRQ (data for the SGRQ was missing in 32 subjects from the reference population sourced from outside of the WRS dataset [10] and from two of the WRS cohort).

Loess lines were fitted to the overall WRS dataset for all 713 subjects who had values for the total score on the SGRQ, plotting the SGRQ versus the post-bronchodilator FEV1/FVC ratio and post-bronchodilator FEV1 \% pred. Loess plots are a form of robust scatter plot smoothing suitable for noisy data [16], providing information on the form of a relationship between variables where it may not be easily described by conventional regression. The loess technique uses a weighted average of a set of data points to fit curves rather than the individual points as in conventional regression. The technique is relatively resistant to outlying values and does not require a prespecified global linear function to be chosen. The degree of smoothing is determined by the width of the window determining the weighted average. Optimal smoothing is obtained by balancing the residual sum of squares for each point against the degree of smoothness.

It was evident that both curves had a point of inflection, where the value of the SGRQ increased from a minimum level, and the value of the SGRQ 4 and 8 units above this point of inflection were examined. Receiver operating characteristic (ROC) curves for two cut-off points for post-bronchodilator FEV1/FVC ratio of 0.7 and 0.65 and the total score on the SGRQ, were fitted to determine discriminatory values, sensitivity and specificity for the SGRQ.

The relationship of the post-bronchodilator FEV1 \% pred, based on equations from the WRS [10], and the loess lines were used to examine the cut-off points for FEV1 \% pred in relation to the GOLD severity cut-off points of 80,50 and $30 \%$ pred.

\section{RESULTS}

\section{Subjects}

There were 2,319 responders to the postal questionnaire, of whom 795 agreed to attend the present authors' research facility for further testing and 713 were able to complete all WRS investigative modules and form the current study group. Subjects in the study group were broadly similar to the 1,606 postal questionnaire responders not in the study group. Subjects in the study group were more likely to be of male sex (54\% versus $44 \%$; $\mathrm{p} \leqslant 0.001)$, smoke tobacco $(13 \%$ versus $10 \% ; \mathrm{p}=0.007)$, have a history of chronic cough $(15 \%$ versus $11 \%$; $p=0.006)$ or breathing trouble $(28 \%$ versus $24 \%$; $=0.04)$ and had a greater mean age (53.9 versus $51.6 \mathrm{yrs} ; \mathrm{p}<0.001)$ than postal questionnaire responders not in the current study group.

\section{Quantile regression prediction equations for SGRQ}

The quantile regression equations for the $95 \%$ and $90 \%$ quantiles for the reference range subjects are $1.53+($ age $\times 0.24)$ and $5.76+($ age $\times 0.096)$, respectively. Using these equations, the $95 \%$ or $90 \%$ quantile is found by inserting the age in years into the equation, for example the $90 \%$ quantile for a $50 \mathrm{yr}$ old is $5.76+(50 \times 0.096)$, which equals 10.56 . The predicted $95 \%$ and $90 \%$ quantiles ranged from 7.9 to 19.0 and 8.3 to 12.8 , respectively, across the age range of $27-74$ yrs. Sex was not an important predictor for these two quantiles. Different 


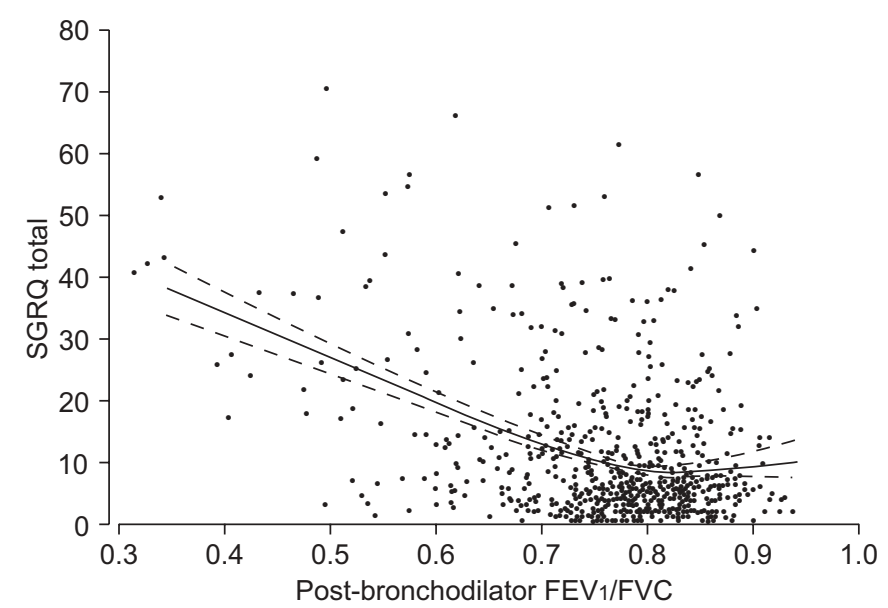

FIGURE 1. The St George's Respiratory Questionnaire (SGRQ) total score versus post-bronchodilator forced expiratory volume in one second (FEV1)/forced vital capacity (FVC) ratio with superimposed loess scatter plot smoother $(-)$ and $90 \%$ confidence interval (- - - ). The loess smoother is a form of regression line using a weighted average of a set of data points at each part of the curve and is robust to outlying values.

coefficients were observed for different quantiles as is allowed by the method of quantile regression.

\section{SGRQ versus post-bronchodilator FEV1/FVC ratio}

Figure 1 shows a scatter plot of the SGRQ total score versus post-bronchodilator FEV1/FVC ratio with the superimposed loess smoothed line and $90 \%$ confidence interval (CI). The point of inflection, the minimum level for the SGRQ, in the loess plot was at a post-bronchodilator FEV1/FVC ratio of $\sim 0.8$. The SGRQ predicted at this value is 8.1 units. A score 4 units higher than this value (SGRQ score 12.1 units), corresponds to a post-bronchodilator FEV1/FVC ratio of 0.71 . A score 8 units higher (SGRQ score 16.1 units), corresponds to a value of the post-bronchodilator FEV1/FVC ratio of 0.65 .

\section{ROC curve analysis}

In total, $119(16.7 \%)$ out of 713 subjects had a postbronchodilator FEV1/FVC ratio of $<0.7$. The area under the ROC curve for the SGRQ for this value of post-bronchodilator
FEV1/FVC ratio was 0.692 , suggesting moderate discrimination of the SGRQ for this cut-off point. In total, 72 (10.1\%) out of 713 subjects had a post-bronchodilator FEV1/FVC ratio of $<0.65$. The area under the ROC curve for the SGRQ for this value of post-bronchodilator $\mathrm{FEV1/FVC} \mathrm{ratio} \mathrm{was} 0.812$, suggesting moderate to good discrimination.

Table 1 shows the sensitivity and specificity values and the corresponding SGRQ total scores for the two post-bronchodilator FEV1/FVC ratio cut-off points of 0.7 and 0.65 . Sensitivity refers to the number of subjects correctly allocated below the cut-off value by the corresponding value of the SGRQ total score divided by the total number of subjects who, in fact, had a ratio below the cut-off point. Specificity refers to the number of subjects correctly allocated above the cut-off point divided by the total number who, in fact, had a ratio above the cut-off point. For example, a SGRQ total score of 12.0 has $66.7 \%$ sensitivity and $70 \%$ specificity for a post-bronchodilator FEV1/ FVC ratio of 0.65 .

Figure 2 shows the full ROC curves for the SGRQ and two values of the post-bronchodilator $\mathrm{FEV1/FVC} \mathrm{ratio.}$

\section{SGRQ versus post-bronchodilator FEV 1}

Figure 3 shows a scatter plot of the SGRQ total score versus post-bronchodilator FEV1 \% pred with superimposed loess smoothed line and $90 \% \mathrm{CI}$. The point of inflection in the loess plot is a post-bronchodilator FEV1 $\sim 100 \%$ pred. The SGRQ predicted at this value is 7.9 units. A score 4 units higher than this value (SGRQ score 11.9 units) corresponds to a value of post-bronchodilator FEV1 $88 \%$ pred. A score 8 units higher (SGRQ score 15.9 units) corresponds to a value of postbronchodilator FEV1 80\% pred.

In figure 3, it can be seen that the relationship between the SGRQ and post-bronchodilator FEV1 \% pred is approximately linear from a FEV1 value of $90 \%$ with a $10 \%$ change in FEV1 associated with a 4 unit change in the SGRQ. The differences in the SGRQ score associated with the GOLD boundaries for severity are shown in table 2 . These data show that there are major differences in the magnitude of change in the SGRQ score between the GOLD severity boundaries of 80,50 and $30 \%$ pred, which define mild, moderate and severe COPD, respectively. In contrast, the magnitude of change in the

TABLE 1 Comparison of the sensitivity and specificity of the SGRQ to identify a low post-bronchodilator FEV $1 / F V C$ ratio using two different post-bronchodilator FEV1/FVC ratio cut-off points

\begin{tabular}{|c|c|c|c|c|c|}
\hline \multicolumn{3}{|c|}{ Post-bronchodilator FEV $1 /$ FVC ratio 0.7} & \multicolumn{3}{|c|}{ Post-bronchodilator $\mathrm{FEV}_{1 / \mathrm{FVC}}$ ratio 0.65} \\
\hline Sensitivity ${ }^{\#}$ & Specificity & SGRQ score & Sensitivity ${ }^{+}$ & Specificity ${ }^{\S}$ & SGRQ score \\
\hline $41(34.5)$ & 534 (89.9) & 21.5 & $32(44.4)$ & $577(90.0)$ & 23.1 \\
\hline $58(48.7)$ & $475(80.0)$ & 13.6 & $43(59.7)$ & $513(80.0)$ & 14.0 \\
\hline $71(59.7)$ & $416(70.0)$ & 9.6 & $48(66.7)$ & $449(70.0)$ & 12.0 \\
\hline $95(79.8)$ & $216(36.4)$ & 4.4 & $58(80.6)$ & $345(53.8)$ & 6.3 \\
\hline $108(90.8)$ & $139(23.4)$ & 2.5 & $65(90.3)$ & $224(34.9)$ & 3.9 \\
\hline
\end{tabular}

Data are presented as $n(\%)$, unless otherwise stated. SGRQ: St George's Respiratory Questionnaire; FEV1: forced expiratory volume in one second; FVC: forced vital capacity. \#: $n=119 ;{ }^{\bullet}: n=594 ;{ }^{+}: n=72 ;{ }^{\varsigma}: n=641$. 


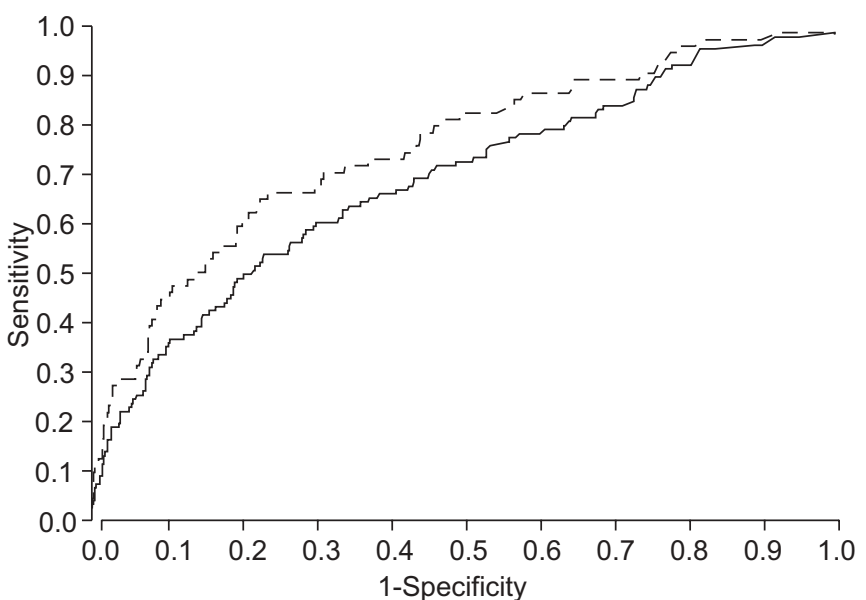

FIGURE 2. Receiver operating characteristic plot showing the ability of the St George's Respiratory Questionnaire to identify a low post-bronchodilator forced

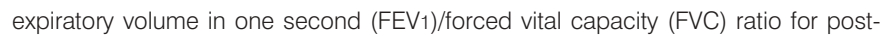
bronchodilator $\mathrm{FEV}_{1} / \mathrm{FVC}$ ratios of $0.65(---)$ and 0.7 ( - ).

SGRQ score between the severity boundaries of 80,60 and $40 \%$ pred (as recommended in asthma guidelines to define mild, moderate and severe disease [17]) were similar, corresponding to approximately twice the clinically important difference for SGRQ (8 units).

\section{DISCUSSION}

The present authors have shown that the suggested boundary of normality for a post-bronchodilator FEV1/FVC ratio of 0.7 for COPD is supported by analysis of the current dataset. This FEV1/FVC value corresponded to a value of the SGRQ score close to the $90 \%$ quantile in the reference range sample representing a clinically important difference in the SGRQ from the nadir of the relationship. The present findings also suggest that the current GOLD cut-off values of 80,50 and $30 \%$ pred to define mild, moderate and severe COPD, respectively,

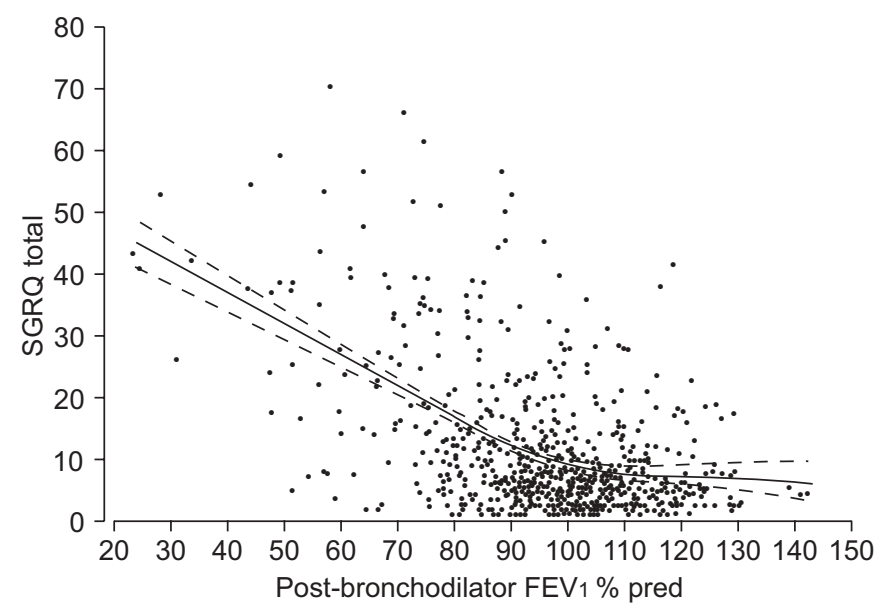

FIGURE 3. St George's Respiratory Questionnaire (SGRQ) versus postbronchodilator forced expiratory volume in one second (FEV1) \% predicted with superimposed loess scatter plot smoother (-) and 90\% confidence interval (----). The loess smoother is a form of regression line using a weighted average of a set of data points at each part of the curve and is robust to outlying values. do not result in a similar magnitude of change in the SGRQ. If the boundaries of severity are to correspond to approximately equal distances on the scale of the SGRQ, boundaries of FEV1 80,60 , and $40 \%$ pred would each correspond to approximately twice the clinically important difference for the SGRQ.

The use of a fixed FEV1/FVC ratio of 0.7 to diagnose COPD has been widely criticised for failing to take account of physiological changes in the value of the ratio with ageing, which can result in overdiagnosis of COPD [3]. The current authors' study group has shown a considerable difference in the prevalence of COPD using a fixed ratio compared with agedetermined predicted values for the FEV1/FVC ratio [9]. In its defence, use of a fixed ratio results in guideline simplicity [1, 18], has been supported by some epidemiological data [19] and values below this have been shown to be predictive of poorer outcomes despite apparent good health [20]. Since, in practical terms, use of a fixed ratio is likely to continue, it is useful to show that this ratio corresponds to clinically significant changes in health status on a validated respiratory questionnaire with reasonable specificity.

Normal reference range values for the SGRQ were derived based on its distribution in healthy nonsmokers, identifying that the $90 \%$ quantile for the SGRQ across the age range in the present reference range group was 12.8 units. This is not dissimilar to values reported in a previous study, although different methods of reporting make direct comparison difficult [8]. The present results also reflect the previously acknowledged effect of age, irrespective of respiratory disease, on health-related quality of life [21]. Using smoothed plots of SGRQ versus post-bronchodilator FEV1/FVC ratio the nadir was 8.1 units for the SGRQ and 0.8 for the post-bronchodilator FEV1/FVC ratio. An increase in the SGRQ score of 4 units, an amount that has been proposed to represent a clinically important difference $[6,8]$, resulted in a value of 12.1 units, which corresponded to a post-bronchodilator FEV1/FVC ratio of 0.7 . An increase in the SGRQ score by a further 4 units corresponded to an FEV1/FVC ratio of 0.65 . Construction of ROC curves identified that a SGRQ value between 12 and 13 units, corresponding to the $90 \%$ quantile in the present reference range sample, had moderate to good discriminative power for a cut-off value for post-bronchodilator FEV1/FVC ratio of 0.7 . Not surprisingly, use of a lower fixed ratio of 0.65 improves the specificity associated with the same SGRQ score, at the cost of reduced sensitivity. Thus, a post-bronchodilator

\begin{tabular}{lcc}
\hline TABLE 2 & $\begin{array}{l}\text { Difference in the SGRQ between GOLD defined } \\
\text { COPD severity boundary points }\end{array}$ \\
$\begin{array}{l}\text { Boundary points in } \\
\text { GOLD severity }\end{array}$ & $\begin{array}{l}\text { Post-bronchodilator } \\
\text { FEV } 1 \text { \% pred }\end{array}$ & $\begin{array}{c}\text { SGRQ unit } \\
\text { difference }\end{array}$ \\
\hline $\begin{array}{l}\text { Mild } \\
\text { Moderate }\end{array}$ & $80-100$ & 8 \\
Severe & $50-80$ & 15 \\
\hline
\end{tabular}

SGRQ: St George's Respiratory Questionnaire; GOLD: Global Initiative for Chronic Obstructive Lung Disease; COPD: chronic obstructive pulmonary disease; FEV 1 : forced expiratory volume in one second; \% pred: \% predicted. 
FEV1/FVC ratio of 0.7 was represented in the present dataset by a clinically important difference in the SGRQ compared with the nadir of the relationship and a value of the SGRQ close to the $90 \%$ quantile in a reference range sample.

The current data was also used to examine the validity of the severity criteria cut-off points for FEV1 espoused by GOLD. Whilst it is known that lower FEV1 values are associated with poorer health status the strength of this correlation is not always that large [21]. Such poor correlations indeed strengthen the case for holistic patient assessment using tools such as quality of life questionnaires and the more recently developed BODE (body mass index, airflow obstruction, dyspnoea and exercise capacity) index [22]. Nonetheless, a FEV1 cut-off point is simple to apply and has been used in guidelines to recommend preferred therapy and in some settings to control access to specific treatments [23]. Whilst the arbitrary nature of cut-off points is acknowledged, it is reassuring that the GOLD severity criteria broadly correlate with the SGRQ, a tool which takes into account the mutlifaceted impact of COPD. However, the present findings suggest that the current cut-off values of FEV1 80, 50 and $30 \%$ pred to define mild, moderate and severe COPD, respectively, do not result in a similar magnitude of changes in SGRQ as the relationship is essentially linear at values $<80 \%$. If the boundaries of severity are to correspond to approximately equal distances on the scale of the SGRQ, boundaries of FEV1 80,60 and $40 \%$ pred would each correspond to about twice the clinically important difference for the SGRQ. The use of the FEV1 80, 60 and 40\% pred cut-off points would also have the advantage of consistency with the grading of severity for asthma [17]. In support of this approach, many older patients with asthma will meet the spirometrically-defined criteria for COPD [24].

There are methodological issues to consider in the current study. There were minor differences in the characteristics of the current study group compared with postal questionnaire responders who did not complete all investigative modules, raising the possibility of selection bias. However, it is unlikely that these differences would systematically affect the relationship between the SGRQ and spirometry. There is a high prevalence of asthma in New Zealand and some of the subjects identified as having COPD from post-bronchodilator spirometry may actually have asthma with incomplete bronchodilator reversibility rather than COPD. In a separate analysis of the WRS data, $\sim 25 \%$ of subjects identified as having COPD had features of asthma and no apparent chronic bronchitis or emphysema [24]. As the proportion of these individuals that have COPD with an asthma-like phenotype and the proportion that have "pure" asthma cannot be determined, individuals with an asthma-like phenotype of airway disease were not excluded from the analysis. There were few subjects in the current study group with severe COPD and, thus, the present analysis primarily applies to those with mild or moderate COPD. The CIs for the relationship between FEV1 and the SGRQ are consequently wider at a low FEV1 and could support a greater, lesser or even nonlinear relationship between FEV1 and the SGRQ in advanced disease. The SGRQ measurements were "noisy" and although the loess scatter plot smoother is useful for examining the underlying pattern of the dataset it may give a spurious impression of precision.
Reducing the degree of smoothing in the algorithm fitting the loess scatter plot smoother may better reflect the noisiness of the data but would reduce the ability to see the overall pattern.

A considerable strength of the present study was that it was a community-based random sample and, therefore, may not be subject to biases created when using diseased populations, such as those attending hospital clinics. The SGRQ is an accepted method of measuring respiratory health quality of life and the definition of a clinically important difference with this instrument means that the analysis reflects associations of clinical importance.

The current authors conclude that, in the diagnosis of chronic obstructive pulmonary disease, the use of a post-bronchodilator forced expiratory volume in one second/forced vital capacity ratio of 0.7 is supported by health-related quality of life measurements. There may be advantages in using postbronchodilator forced expiratory volume in one second cut-off points of 80,60 and $40 \%$ predicted for the classification of mild, moderate and severe COPD, respectively, similar to the approach recommended for asthma.

\section{REFERENCES}

1 Global strategy for the diagnosis, management and prevention of COPD. Global Initiative for Chronic Obstructive Lung Disease (GOLD) 2008. www.goldcopd. org Last updated: July 2008. Last accessed: February 2009.

2 Pellegrino R, Viegi G, Brusasco V, et al. Interpretative strategies for lung function tests. Eur Respir J 2005; 26: 948 968.

3 Hardie JA, Buist AS, Vollmer WM, Ellingsen I, Bakke PS, Morkve O. Risk of over-diagnosis of COPD in asymptomatic elderly never-smokers. Eur Respir J 2002; 20: 11171122.

4 Mannino DM. Defining chronic obstructive pulmonary disease... and the elephant in the room. Eur Respir J 2007; 30: 189-190.

5 Hankinson J, Odencrantz J, Fedan K. Spirometric reference values from a sample of the general US population. Am J Respir Crit Care Med 1999; 159: 179-187.

6 Jones PW. St George's Respiratory Questionnaire: MCID. COPD 2005; 2: 75-79.

7 Maleki-Yasdi M, Lewczuk CK, Haddon JM, Choudry N, Ryan N. Early detection and impaired quality of life in COPD GOLD stage 0: a pilot study. COPD 2007; 4: 313-320.

8 Ferrer M, Villasante C, Alonso J, et al. Interpretation of quality of life scores from the St George's Respiratory Questionnaire. Eur Respir J 2002; 19: 405-413.

9 Shirtcliffe P, Weatherall M, Marsh S, et al. COPD prevalence in a random population survey: a matter of definition. Eur Respir J 2007; 30: 232-239.

10 Marsh S, Aldington S, Williams M, et al. Complete reference ranges for pulmonary function tests from a single New Zealand population. N Z Med J 2006; 119: U2281.

11 Standardization of spirometry, 1994 update. American Thoracic Society. Am J Respir Crit Care Med 1995; 152: 11071136.

12 Jones P. Quality of life measurement for patients with diseases of the airways. Thorax 1991; 46: 676-682. 
13 Jones PW, Quirk FH, Baveystock CM. The St George's Respiratory Questionnaire. Respir Med 1991; 85: Suppl. B, 25-31.

14 Jones PW, Quirk FH, Baveystock CM, Littlejohns P. A selfcomplete measure of health status for chronic airflow limitation: the St George's Respiratory Questionnaire. Am Rev Respir Dis 1992; 145: 1321-1327.

15 Koenker $\mathrm{R}$. The $\mathrm{R}$ project for statistical computing. $\mathrm{R}$ package version 4/06/2007. www.r-project.org Date last updated: October 20, 2008. Last accessed: February 2009.

16 Freund RJ, Littell RC. Curve fitting. In: Freund RJ, Littell RC, eds. SAS System for Regression. 3rd Edn. Cary, SAS Institute, 2000; pp. 150-155.

17 Global Initiative for Asthma. Global Strategy for Asthma Management and Prevention 2008. www.ginasthma.org Date last updated: 2008. Date last accessed: February 2009.

18 Calverley P, Kerstjens H. Pro/con editorials: the GOLD classification has advanced understanding of COPD. Am J Respir Crit Care Med 2004; 170: 211-214.
19 Johannessen A, Lehmann S, Omenaas ER, Eide E, Bakke PS, Gulsvik A. Post-bronchodilator spirometry reference values in adults and implications for disease management. Am J Respir Crit Care Med 2006; 173: 1316-1325.

20 Mannino DM, Buist AS, Vollmer WM. Chronic obstructive pulmonary disease in the older adult: what defines abnormal lung function? Thorax 2007; 62: 237-241.

21 Jones PW. Health status measurement in chronic obstructive pulmonary disease. Thorax 2001; 56: 880-887.

22 Celli BR, Cote CG, Marin JM, et al. The body-mass index, airflow obstruction, dyspnea and exercise capacity index in chronic obstructive pulmonary disease. N Engl J Med 2004; 350: 1005-1012.

23 Jones D. Long-acting inhaled bronchodilators for COPD lack of logic continues. N Z Med J 2005; 118: U1669.

24 Marsh SE, Travers J, Weatherall M, et al. Proportional classifications of COPD phenotypes. Thorax 2008; 63: 761-767. 